\title{
APPLIED RESEARCH ON TECHNOLOGIES OF DESIGN FOR MASS CUSTOMISATION
}

\author{
Qiaoxiang Gu, Guoning Qi, Shaohui Su, Yujun Lu \\ Institute of Contemporary Manufacturing Eng., Zhejiang Univ., Hangzhou, China ; Email: \\ guqiaoxiang@163.com
}

\begin{abstract}
Utilizing the rotor and cylinder of the steam turbine as examples, the key technologies of design for mass customization (DFMC) have been researched. Out of this research, an applied system of design for mass customisation has been assembled. This system consists of several modules, including the system integrated Smart Team PDM, with a coding module and tabular layouts of article characteristics module (both developed by the research team), and Solid Edge CAD software. The system provides facilities for designing customisation products that satisfy individual customers' needs, and has been applied in the design of the steam turbine.
\end{abstract}

Key words: Mass customisation; product master model; product master structure

\section{INTRODUCTION}

Mass customization has recently received much attention from both industry and academia. It aims to deliver an increasing product variety to satisfy diverse customer needs while maintaining near mass production efficiency[1,2].

Du et al. describe how an architecture of product families contributes to the generation of families of products efficiently[4]. Jianxin Jiao et al. propose a triple-view scheme for modelling single products, including function view, technical view and structural view[5]. Sehyun et al. describe the parametric modelling process for machine tools, and propose a framework which parametrically models a machine tool assembly based on a design expert system[6].

The research team's framework of technology for mass customisation consists of four parts, of which design for mass customisation (DFMC) is the

This project is supported by the National Natural Science Foundation of China under the grant No. 50575204 and 60374057

Please use the following format when citing this chapter:

Gu, Qiaoxiang, Qi, Guoning, Su, Shaohui, Lu, Yujun, 2006, in International Federation for Information Processing (IFIP), Volume 207, Knowledge Enterprise: Intelligent Strategies In Product Design, Manufacturing, and Management, eds. K. Wang, Kovacs G., Wozny M., Fang M., (Boston: Springer), pp. 1030-1036. 
key part[3]. Under the direction of the technologies for modularisation and tabular layouts of article characteristics (TLAC module), the system constructs the product master models (PMM) of variant products and establishes the product master structure (PMS). On the basis of the PMM and PMS, using the configuration design and variant design, the engineers can design the individual product to satisfy customer needs.

\section{FRAMEWORK OF THE SYSTEM FOR DFMC}

The framework of the system for DFMC is a three-layer architecture, consisting of the user layer, logic layer and database (see Figure 1). In the system, Smart Team PDM was chosen as the user platform, and integrated with the coding module, tabular layouts of article characteristics module and the Solid Edge CAD system. The members of the project developed the coding module and tabular layouts of article characteristics module. The coding module is the core and base of DFMC. Although Smart Team PDM includes a code generation function, this does not meet the requirements of the DFMC. Therefore, we developed the coding module for DFMC.

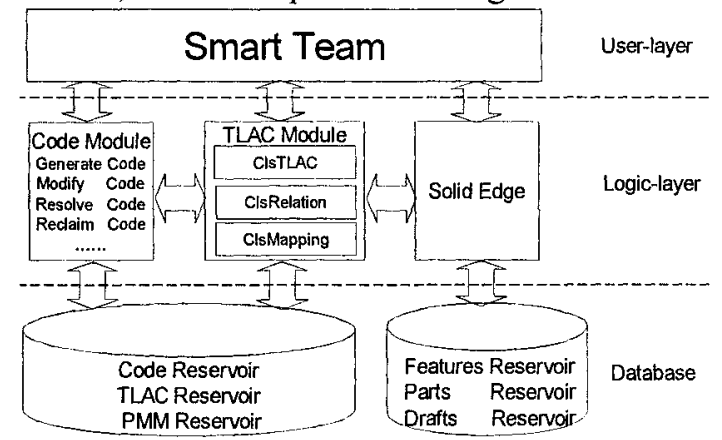

Figure 1. The framework of the system for DFMC

Using the tabular layouts of article characteristics module, the designers can construct product master models of variant products and generate variant products, which meet customer requirements. The module comprises the class of TLAC (ClsTLAC), the class of relations of the TLACs (ClsRelation) and the class of mapping relations between TLAC and CAD (ClsMapping). This module provides the facility to establish the TLAC of the product master models and to create the variant products that meet customer requirements. 


\section{THE LOGIC STRUCTURE OF THE DATABASE}

The database of DFMC consists of code reservoir, features reservoir, PMM reservoir, TLAC reservoir, parts reservoir, drafts reservoir, etc. The system uses the same code module in order to ensure the consistency of data in the development of the product. In this way, all products data and documents are searched and utilized effectively. The logic structure of the database is illustrated in Figure 2.

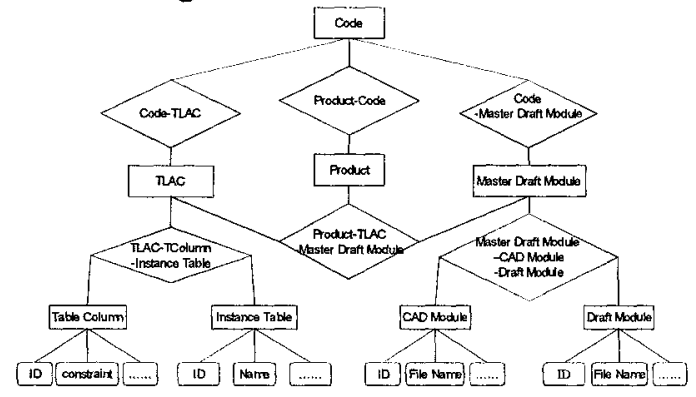

Figure 2. The logic structure of the database

\section{THE KEY TECHNOLOGIES OF DFMC}

DFMC includes development for mass customization and design for mass customization [3]. The key technologies of DFMC consist of code for DFMC, modularisation, product modelling, product master structure and the product configuration, and so on. Connected with the design for the cylinder and rotor of the steam turbine, how to apply the key technologies will be researched in the next paragraphs.

\subsection{The code for DFMC}

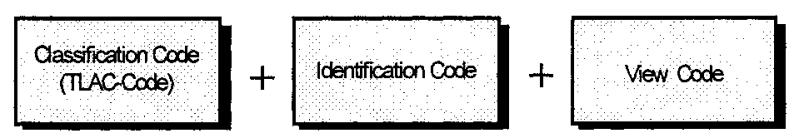

Figure 3. The code for DFMC

The code module is the base and core of the system for DFMC. The code for DFMC is composed of the classification code, identification code and view code (Figure 3 ). These can be used in combination.

The classification code (TLAC code) is used to manage the part family in the product family. It can adopt the membership code or classification code. 
The identification code is unique in the life cycle of the parts, and it adopts a sequential code.

\subsection{Modularization technology}

On the basis of overall analysis and research into customer needs, using the modularisation technology, the designers developed an independent function module, which can be used to assemble the complete product family. This module is the key to product configuration design and variant design. It can reduce the internal diversification of products and enhance the exterior diversification of products.

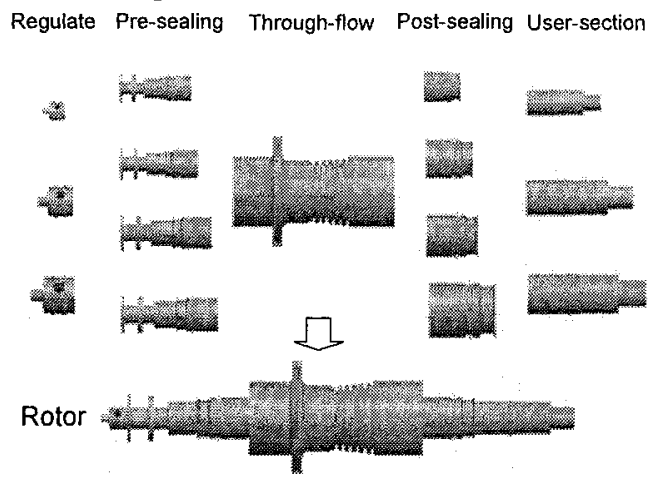

Figure 4. Theory for module design the rotor of industrial steam turbine

Under the direction of the principles of modularization, the rotor of the steam turbine can be divided into five sections in logic, which are regulate, pre-sealing, through-flow, post-sealing and user sections. With the exception of the through-flow section, the sections are designed for standardisation modules, which are in a numerical system. According to customer needs, the engineers design the model of the through-flow section. The designers can then choose the corresponding models from the series models of the others sections, and assemble the rotor model quickly.

\subsection{Product master model}

The product master model contains the geometric and non-geometric information of product, which is used to for the full life cycle of the product. The system induces the TLAC to create the product master model $[7,8]$. Inputting a set of parameters that reflect customer needs into the TLAC of the PMM, we can generate the variant products automatically.

Depending on the product, there are two kinds of variant, dimension variant and structure variant. Gu et al. present how to construct the TLAC of 
the dimension variant [8]. We now discuss how to construct the structure variant part's TLAC.

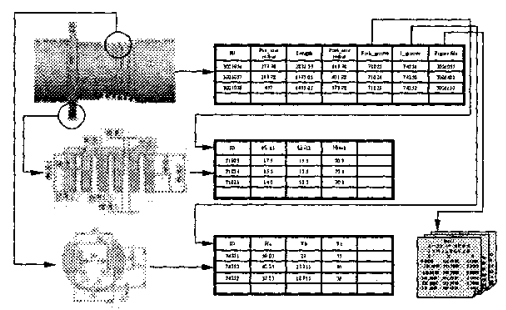

Figure 5. TLAC of through-flow section

The through-flow section of the steam turbine is a typical structure variant part. From customer needs, the designers determine the model parameters. The through-flow section model includes the steam-seal groove and lamina-foot groove. Except for the location dimension of the model, the other dimensions are structure variant. The structure variant items consist of type of steam-seal groove and location, type of lamina-foot groove and location. These are saved in data files. The TLAC of the model saves the index of the data files (Figure 5).

\subsection{Product master structure}

The product master structure (PMS) describes the configurable product family, which contains the standardization modules. Based on the customer needs, the designer can determine the customisation product's structure from the PMS.

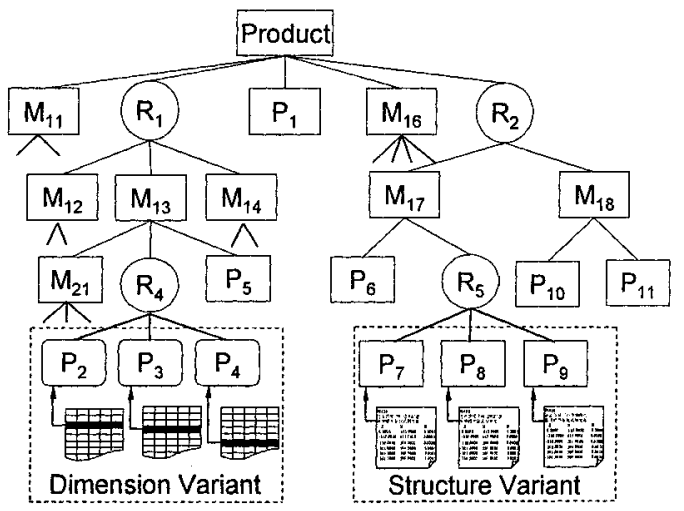

Figure 6. Product Master Structure

When we construct the PMS, the hierarchical structure of the basic modules should be constructed, and then added to the selected modules (Figure 6). In the PMS for product family, it used the rules to choose the 
selected modules. Here the configuration rules were described by EventCondition-Action rule. In the PMS, it includes the dimension and structure variant module, which were mentioned in subsection 4.3.

Definition: PMS is a pair, PMS:: $=(\{$ Modules $\},\{$ ECA rules $\})$, Where:

\{Modules\} represents a set of modules. \{Module\}::= (ID, Name, Parameter, Description), the elements of set describe the identification number, name, feature-parameter and description of the module.

\{ECA rules\} is a set of configuration rules. R::=(ID, Event, Condition, Action). Where the elements of set represent the identification number, trigger, precondition and conclusion of the configuration rules.

\subsection{Product configuration design}

According to the customer needs, the product structure and parts of customisation product can be gained from the Product Configuration Design. We can use Solutions $=F$ (PMS, Requirements) to describe the process of configuration. Where $\mathrm{F}$ is the solution process; PMS describes product master structure; Requirements represents customer needs; Solutions describes the configuration scheme, which satisfied with customers.

To customize the steam turbine, customer needs include the type of the steam turbine, the pressure and temperature of inlet steam, the pressure and temperature of exhaust steam, the output power and rotate speed of working machine, and so on. Surely, it is an iterative process to solve of the product configuration. To the steam turbine, which is ETO, it is impossible to depend on the computer to accomplish the configuration completely, and sometimes need the designer to join.

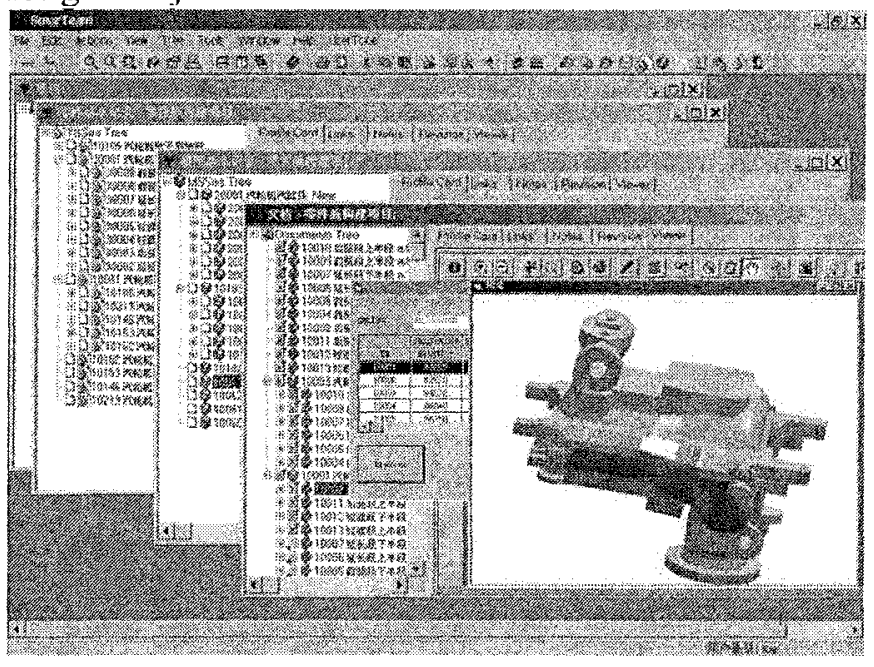

Figure 7. Application Instance 


\section{CASE STUDY}

The steam turbine is customised completely by customer needs. If designers induce DFMC to design it, it will reduce parts number obviously and enhance the design efficiency. The cylinder of steam turbine includes pre-section, decompression-section, transition-section, prolong-section and exhaust-steam-section. According to the through-flow section, these modules can be assembled to the cylinder by rules.

Figure 7 provides a rotor instance. The cylinder includes of pre-section, transition-section, prolong-section and exhaust-steam-section.

\section{CONCLUSIONS}

Using the system for DFMC to develop the steam turbine, it not only enhances the design efficiency, but also reduces the cost of production. Further, in order to exploit the effect of DFMC, it should be combined with management for mass customization and manufacture for mass customization.

\section{REFERENCES}

1. Pine II, B.J.,(1993),Mass Customization: The New Frontier in Business Competition, Harvard Business School Press (ISBN: 0875849466).

2. Tseng, M. M., Jiao, J.,(1996),Design for mass customization,Annals of the CIRP, 45, pp. 153-156.

3. Qi Guoning, Gu Xinjian, Tan Janrong,(2003), Mass Customization Technologies and Development, china machine press (ISBN: 7-111-13110-x).

4. Xuehong Du, Mitchell M.Tseng, Jianxin Jiao, (2001), Product Families for Mass Customization-Understanding the architecture,The Customer Centric Enterprise Advances in Mass Customization and Personalization, Springer-Verlag Berlin Heidelberg New York. (ISBN: 3-540-02492-1)

5. Jianxin Jiao, Mitchell M.Tseng, Vincent G. Duffy, (1998), Product Family Modeling for Mass Customization, Computers ind. Engng, Vol.35, Nos 3-4, pp.495-498.

6. Sehyun Myung, Soonhung Han, (2001),Knowledge-based parametric design mechanical products based on configuration design method,Expert Systems with Applications 21. pp.99-107.

7. Lu Yunjun, Yu Junhe, Qi Guoning, (2003), Product Variant Design Based on Tabular Layouts of Article Characteristics, Computer Integrated Manufacturing Systems, Vol. 9 No. 10 , pp.840-844.

8. Gu Qiaoxiang, Su Shaohui, Yu Junhe, (2004), Research on Product Variant Design Based on the Tabular Layouts of Article Characteristics, China Mechanical Engineering, Vol.15 No.19, pp.1713-1716. 